\title{
Hardware- and Software-in-the-loop Simulation for Parameterizing the Model and Control of Synchronous Condenser
}

Nguyen, Ha Thi; Yang, Guangya; Nielsen, Arne Hejde; Jensen, Peter Højgaard

Published in:

IEEE Transactions on Sustainable Energy

Link to article, DOI:

10.1109/TSTE.2019.2913471

Publication date:

2019

Document Version

Peer reviewed version

Link back to DTU Orbit

Citation (APA):

Nguyen, H. T., Yang, G., Nielsen, A. H., \& Jensen, P. H. (2019). Hardware- and Software-in-the-loop Simulation for Parameterizing the Model and Control of Synchronous Condenser. IEEE Transactions on Sustainable Energy, 10(3), 1593-1602. https://doi.org/10.1109/TSTE.2019.2913471

\section{General rights}

Copyright and moral rights for the publications made accessible in the public portal are retained by the authors and/or other copyright owners and it is a condition of accessing publications that users recognise and abide by the legal requirements associated with these rights.

- Users may download and print one copy of any publication from the public portal for the purpose of private study or research.

- You may not further distribute the material or use it for any profit-making activity or commercial gain

- You may freely distribute the URL identifying the publication in the public portal 


\title{
Hardware- and Software-in-the-loop Simulation for Parameterizing the Model and Control of Synchronous Condensers
}

\author{
Ha Thi Nguyen, Student Member, IEEE, Guangya Yang, Senior Member, IEEE, Arne Hejde \\ Nielsen, Senior Member, IEEE, and Peter Højgaard Jensen
}

\begin{abstract}
The rapid increase of converter-based generation in the grid causes significant changes in the system dynamic characteristics. These changes require verification and testing of the reliability, optimization, and functionality of power system elements and controllers, which become more important for guaranteeing a secure operation. This paper first introduces a hardware-in-the-loop (HiL) setup of an automatic voltage regulator (AVR) control system and then proposes a softwarein-the-loop (SiL) setup for the parameterization of an IEEE standard AVR/excitation system model and a power oscillation damping (POD) controller of synchronous condenser. The AVR hardware is interfaced with a simulated grid in real-time digital simulator (RTDS) using EMT simulation to evaluate its control functions. Parameter optimization for the POD and the AVR simulation model by SiL simulation is implemented in a closed loop between RTDS and Matlab/Simulink interfaced through OPC to satisfy the optimization objectives. By tests executed on the future Western Danish power system, parameter optimization of the POD and IEEE standard AVR model, and the function of AVR hardware are successfully implemented and verified.
\end{abstract}

Index Terms-Automatic voltage regulator, hardware-inthe-loop, parameter optimization, power oscillation damping, software-in-the-loop.

\section{INTRODUCTION}

$\mathbf{T}$ HE major energy transition in the stepwise replacement of synchronous-machine-based systems by renewablebased systems renders the system operation more challenging due to the different physical characteristics and interaction with the grid of converter-interfaced components. Traditional power plants are usually immense synchronous generators that are capable of providing ancillary services for balancing and transient characteristics. Conversely, the majority of renewable energy generators are interfaced to the grid via power electronic converters that have different characteristics and are often controlled in such a way that they are independent of the system frequency. In addition, high importing HVDC interconnections are also expected to replace the traditional generation. These factors have introduced many challenges to secure operation and forced power systems to operate in low inertia conditions. The combination of reduced system inertia and intermittent renewable sources also causes considerable

H. T. Nguyen, G. Y. Yang and A. H. Nielsen are with the Center for Electric Power and Energy, Department of Electrical Engineering, Technical University of Denmark, 2800 Kongens Lyngby, Denmark (e-mail: (thangu, gyy, ahn)@elektro.dtu.dk, nthadht@gmail.com)

P. H. Jensen is with Energy Automation, Siemens A/S, 2750 Ballerup, Denmark (e-mail: peter_hoejgaard.jensen@siemens.com) changes in the system dynamic characteristics. Oscillatory issues from low inertia operating conditions, poor frequency control support and weak interconnections among power systems are more significant. In [1], [2], different frequency range oscillations in power systems with high shares of renewable sources are found in Texas and China power systems. These oscillations are caused by converter-based generations connected to weak power systems [3].

From the above analysis, low inertia systems are inevitable in the green energy era, which introduces new stability issues and requirements for controls. This approach requires innovative solutions for verification and testing of the reliability, functionality and optimization of power system elements and controllers to guarantee a secure operation.

To address these issues, hardware-in-the-loop (HiL) tests for supplementary control have been introduced. In HiL simulation, the interface between the simulation system and the test component is being emulated such that the inputs to the component seem to be derived from a real system and the control signals from the component cause corresponding actions in the model. In [4], a real-time HiL test for an excitation control system of a synchronous generator using external stabilizing signals to improve oscillation damping is investigated. A control HiL for automatic voltage regulator is implemented in [5] to evaluate the control functions during steady-state and dynamic conditions. HiL simulation for superconducting DC power transmission system, which investigated the stability of a superconducting DC cable system in steady state and transient conditions, is presented in [6]. Reference [7] implements an HiL test for protective relaying control adjustments and training tools using the reduced Colombian power system. A HiL of distance protection relay of synchronous condensers under unbalanced faults is developed in [8], while a hardware simulation of a static VAR compensator for load compensation is designed and implemented in [9]. Furthermore, a realtime hardware and SiL simulation is developed to evaluate the functionality of decentralized distribution network control architecture [10]. A control HiL simulation of a permanent magnet synchronous motor in healthy and faulty conditions is proposed in [11]. The simulation proves that control HiL is a safe and relatively inexpensive environment for developing fault detection and diagnostic algorithms.

Parameter optimization of controllers is another approach to ensure the secure operation for modern power systems. Control engineers are concerned with the efficient implementation 


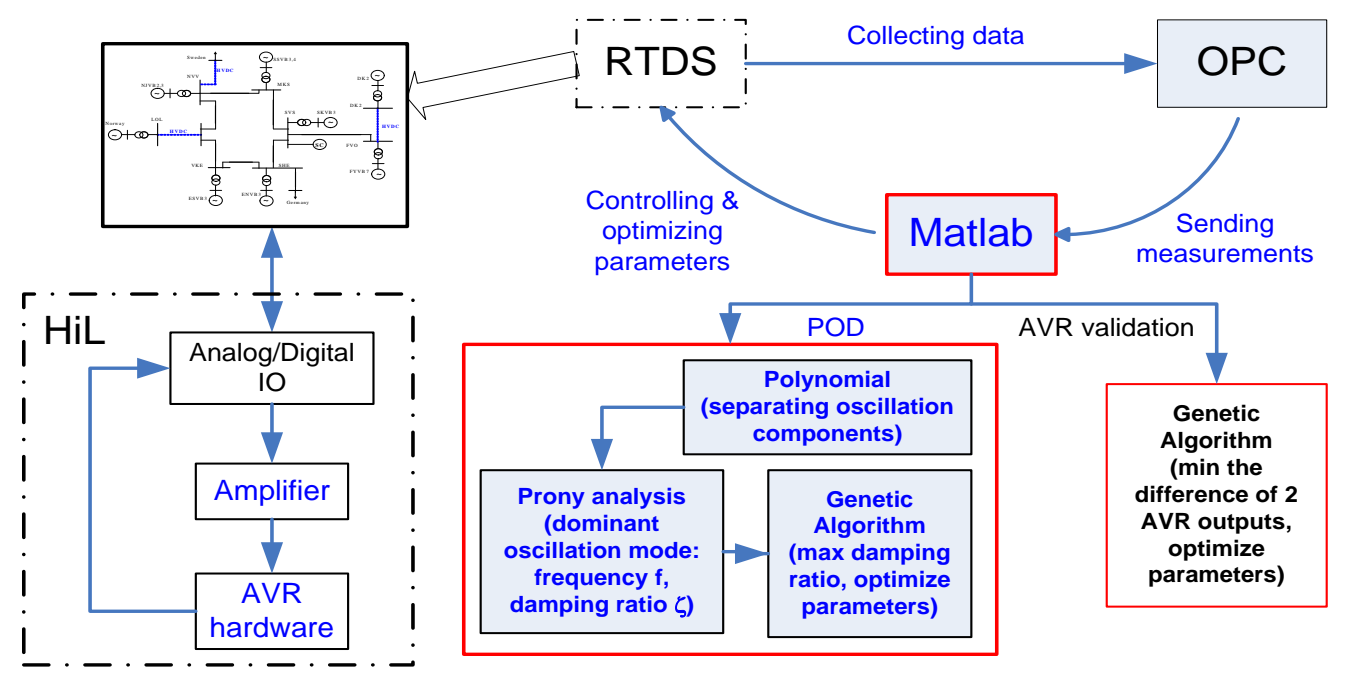

Fig. 1. System arrangement of HiL and SiL simulation.

of parameter optimization to maximize their testing. In the literature, several researchers have implemented optimization algorithms for determining an optimal parameter set of controllers using the Matlab program. Tuning parameter for a PID of an AVR control system [12], [13] or a PSS [14]-[17] for an $\mathrm{SG}$ in synchronous-machine-based systems is well researched using different algorithms. A new controller that interacts between the active power and reactive power channels of SCs to improve primary frequency control is investigated in [18]. This control method is able to assist the governor response by reactive power modulation to improve the frequency nadir and settling time. However, this method does not adequately deal with the oscillation damping and mention about the parameter optimization of the proposed controller. In [19], a genetic algorithm (GA) optimization is applied for tuning control parameters of the proportional integral controller subject to the $H_{\infty}$ constraints in terms of linear matrix inequalities of the state-space system model of the three-area power system. Reference [20] proposes a tuning strategy for a robust pulse width modulated series compensator damping controller that is based on an augmented Lagrangian particle swarm optimization which satisfies the multiple $H_{\infty}$ performance criteria for FACTs controller. A PSS parameter optimization using a trajectory sensitivity approach for a multi-machine power system is examined in [21]. However, parameter optimization by the Matlab simulation using RMS simulation based on the system-theoretic approaches either linear model or other algorithms, which depends on the initial operating point with several simplifications, e.g. no consideration of discrete control behaviors like limiters, saturation. As a result, it is limited for evaluating the control system performance when it reaches the limits or discrete functions during disturbances. A gradientbased nonlinear parameter optimization of FACTs devices via Real-Time Digital Simulator (RTDS) is investigated in [22], [23]. However, the research is limited due to a lack of optimization algorithm implementation support in the RTDS.

The paper introduces a HiL setup for an automatic voltage regulator (AVR) and proposes software-in-the-loop (SiL) sim- ulation for parameterization of a power oscillation damping (POD) controller incorporating synchronous condenser (SC) and an IEEE standard AVR model to evaluate the functionality and the optimization of these elements, as a part of the SCAPP project [24]. The system includes a simulated grid in RTDS interfaced with AVR hardware, which is employed in the field and differs from the IEEE model. Furthermore, a SiL among the RTDS, Matlab, and OPC using real-time EMT simulation for optimizing the parameter of the control and the model is proposed. The proposed method uses Prony analysis to extract the system information based on measurement data which is applicable in the field. On top of it, the proposed method tends to apply to converter-based systems where the mechanism and characteristics are completely different to the synchronousmachine-based systems, and the POD and AVR are focused on the SCs.

The paper aims to optimize parameters of POD control for damping oscillation and enhancing frequency stability and those of the IEEE standard AVR model to achieve a behavior that is similar to the AVR hardware and test the functionality of an AVR controller in hardware before commissioning the system for field demonstrations. From the test executed with the future Western Danish power system [25], the parameterization of the POD and the AVR model and the functionality of AVR features are successfully implemented and verified.

The remaining part of the paper is organized as follows. The system arrangement is generally introduced in Section II. Section III presents the AVR implemented in hardware which includes different limiters of SC. A SiL of the POD and PID parameter optimization is investigated in section IV. In section $\mathrm{V}$, the different test results of the actual AVR system and parameterization based on SiL simulation are analyzed and verified. Some important conclusions are finally drawn in section VI.

\section{SySTEM ARRANGEMENT}

The whole future Western Danish power system is modeled in RSCAD and run in real time at RTDS platform. This 
system is interfaced with an AVR system in hardware through digital/analog inputs/outputs. In addition, a SiL simulation for parameterization is implemented with a communication of RTDS, Matlab, and OPC. The whole system arrangement is shown in Fig. 1.

The RTDS is a fully digital power system simulator operating in real time, which can solve the power system equations fast enough (typically $50 \mu \mathrm{s}$ time step) to continuously produce output conditions that realistically represent conditions in the real network [26]. Therefore, it provides a solid framework for testing, development, and evaluating the performance of power system controllers under a similar condition to real environment in practice.

Fig. 2 shows the overall procedure of optimization process with SiL and HiL in sequences, where the entire optimization process is carried out in many successive runs until the parameter set $(x)$ satisfies the user-defined stopping criteria.

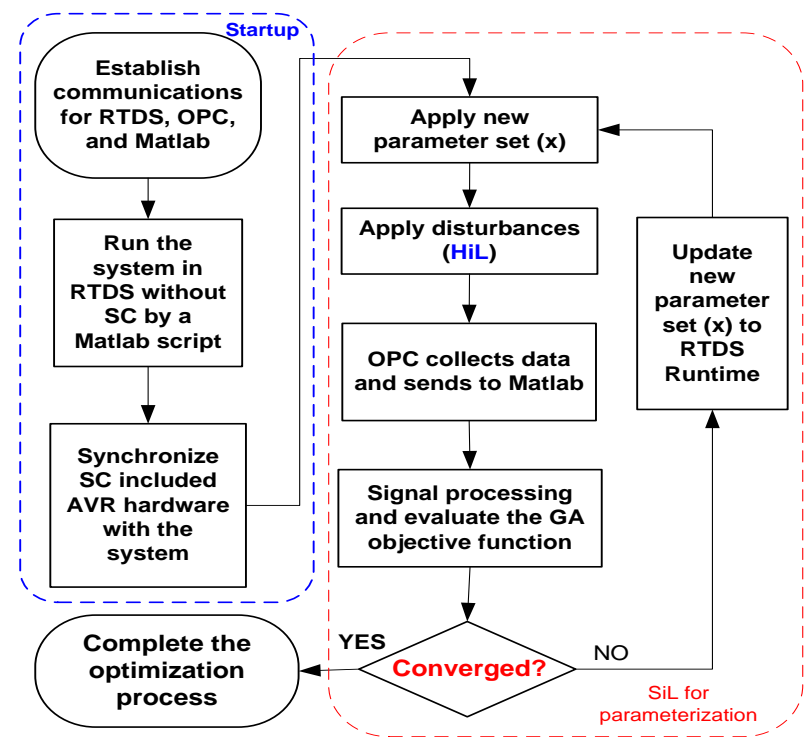

Fig. 2. The overall procedure of optimization process with SiL and HiL.

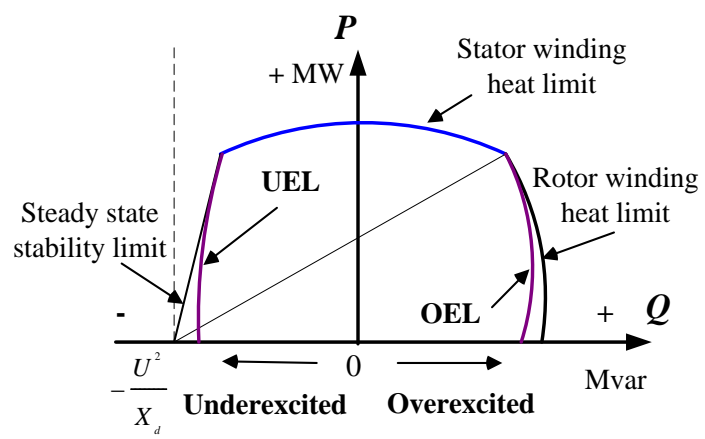

Fig. 3. The PQ capability diagram of the synchronous generator.

\section{AVR HARDWARE-IN-THE-LOOP SETUP}

Fig. 3 shows a PQ capability diagram of the synchronous generator, which is considered to be a thermal diagram of the machine. The stator current limit represents the stator winding isotherm, the field current limit describes the rotor winding isotherm, and the limit in capacitive regime conditions acts for the isotherm of the frontal teeth. Regarding the synchronous condenser, the field current limit, the stator current limit, and the flux limit have a key role to guarantee operation in the steady-state stability margin. These limiters are embedded in the AVR hardware as the thermal protective functions, namely, underexcitation limiter (UEL), overexcitation limiter (OEL), and stator current limiter (SCL). The UEL prevents the synchronous condenser from operating below the end core limits and the steady-state stability limit; the OEL prevents the synchronous condenser from operating above the rotor winding heat limit; and the SCL protects the stator winding from operating over the heat limit. These limiters enable the SC to operate in the maximum transient to support the power system during disturbances while ensuring that SC operation remains within allowable regions, as indicated in Fig. 3.

The AVR model implemented in the HiL platform is based on PID control that is modified from the AVR model of the IEEE AC7B excitation system, as shown in Fig. 4 [27]. The AVR detects the terminal voltage of SC through voltage compensator and transforms the signal to $V_{C}$, and then compares with the reference value $V_{\text {ref }}$. The difference $e$ between these two values works as the input for PID control to create the AVR output $V_{R}$ :

$$
V_{R}=K_{P} e+K_{I} \int_{0}^{t} e \mathrm{~d} t+K_{D} \frac{\mathrm{d} e}{\mathrm{~d} t}
$$

where $K_{P}, K_{I}$, and $K_{D}$ are the proportion, integral, and differential gains of the PID control, respectively.

Whenever a difference between $V_{C}$ and $V_{\text {ref }}$ is detected, PID control immediately reacts to correct the deviation. The higher is $K_{P}$, the faster is the correction. However, a large $K_{P}$ can cause a large oscillation in the output. The static error of the terminal voltage is eliminated by the integral function. The lower is $K_{I}$, the smaller is the overshoot obtained by the output; however, the system may experience more oscillations during the dynamic period. The differential function improves the disturbance rejection ability but may carry the noise of the input to the output. A low-pass filter $\left(\frac{1}{1+s T_{D}}\right)$ is usually added for the derivative term to reduce the noise.

The value of $V_{R}$ is converted to a percentage as the AVR set point sending to the frequency converter of the excitation system as shown in Fig. 4. The frequency converter, exciter, and diode bridge parts are implemented in RTDS with SC and the grid models. An AVR simulation based on the IEEE AC7B model with the parameter set of PID controller is optimized by an GA objective to build an AVR simulation having a similar behaviour to the AVR hardware.

Fig. 5 presents the configuration diagram of the system interfaced with the hardware-in-the-loop test of the AVR. The entire grid and SC model without the AVR system are simulated in RTDS with the dashed blue boundary, while the AVR system is implemented in the hardware with the dashed red boundary. The RTDS sends the three-phase current and voltage of the generator side, the excitation current and voltage, and the busbar frequency and voltage of the grid 


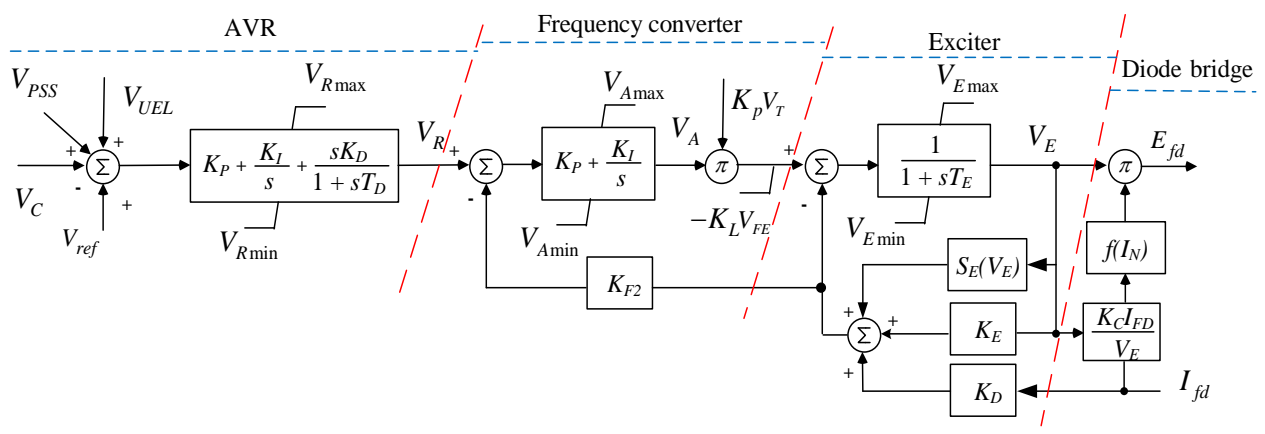

Fig. 4. IEEE AC7B excitation system [27].

side to the hardware platform, whereas the hardware platform transfers the AVR setpoint (AVR SP) and ON GRID signal to the RTDS [5] as shown in Fig. 5(a).

The entire SC system, including the AVR controller, is programmed in VisSim software in PC1 for the startup procedure of $\mathrm{SC}$, whereas the AVR hardware is programmed in the programmable logic controller (PLC) S7-400 via WinCC, which is located at PC2, as shown in Fig. 5(a). The main components of the hardware platform consist of two PLCs S7-1500 and S7-400H and measurement transformers. S71500 is responsible for the measurement data and computation of signals from transformers, while $\mathrm{S} 7-400 \mathrm{H}$ is in charge of AVR control functions. Three-phase $100 \mathrm{VAC}$ voltage and 1 AAC current outputs from the OMICRON power amplifier are sent directly to the measurement transformers. These voltage and current signals transfer to S7-1500; after storing and processing these signals, they are sent to $57-400 \mathrm{H}$, where the AVR is programmed.

Regarding RTDS interface, RTDS can receive and send their analog and digital signals to HiL through Giga Transceiver I/O cards (GTIO) that include Giga Transceiver Analog Input/Output cards (GTAI/GTAO) and Giga Transceiver Digital Input/Output cards (GTDI/GTDO). With terminal voltage and current outputs of SC, the analog input of the external device requires other ranges which are $1 \mathrm{~A}$ for the current and 100 $\mathrm{V}$ for the voltage corresponding to their nominal values. A power amplifier is therefore used to satisfy the HiL required values as shown in Fig. 5(b). Meanwhile, the excitation current and voltage, as well as the frequency and voltage of busbar through scaling ratios inside of GTAO card are sent directly to the hardware. The ON GRID signal is sent to RTDS from the hardware through a GTDI card and AVR setpoint from the hardware is sent to RTDS via a GTAI card as shown in Fig. $5(b)$.

After executing the SC start sequences, the SC is synchronized with the system. Over-excitation, under-excitation conditions, and a load increase disturbance are implemented to verify the performance of AVR hardware and simulation with parameter optimization.

\section{A. Underexcitation limiter implementation}

When the SC becomes underexcited to the point that the negative reactive power may fall below the SC capability limit, the UEL will react by producing a positive error signal that

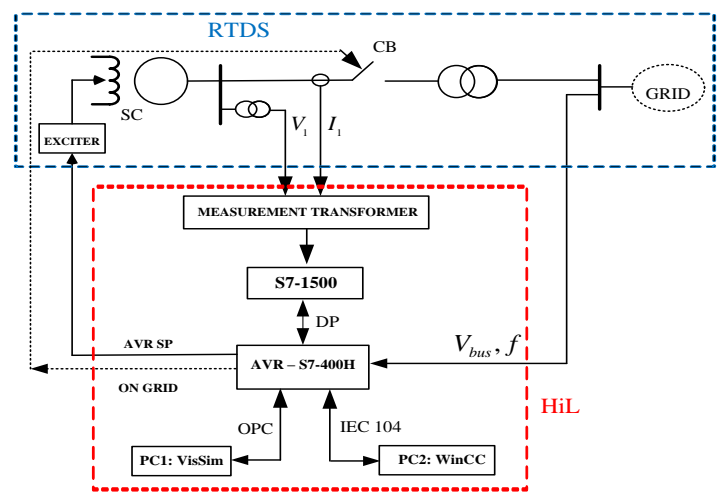

(a) The system diagram with AVR HiL.

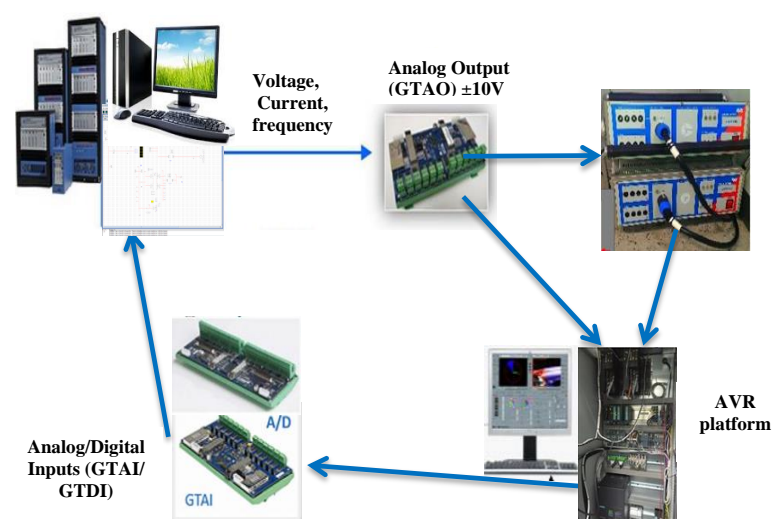

(b) AVR hardware testing setup.

Fig. 5. Hardware in the loop testing setup for AVR system.

will be supplied to the AVR to require the AVR to increase the output voltage by increasing the voltage setpoint until the SC operating point increases above the UEL limit line characteristics [28], [29]. One important function of the UEL is to prevent SCs from operating beyond their steady-state stability limits, as shown in Fig. 3. The underexcited operation is limited to a point, where a small minimum excitation level is required for stability reasons.

Fig. 6 shows the UEL limiter that is implemented in the PLC. The function is based on the measured active power, reactive power, and terminal voltage of the SC. The block $Q(P)$ symbolizes a look-up table that converts $\mathrm{P}$ values to $\mathrm{Q}$ values. Whenever the measured reactive power $Q$ is greater 
than the output of $Q(P)$, a difference is sent to a PI controller. Additionally, the output of the PI controller can be set to zero using the enable signal.

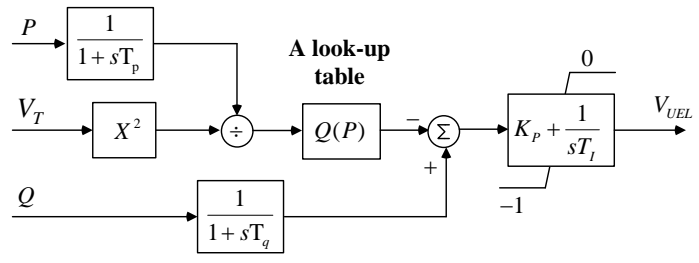

Fig. 6. UEL limiter.

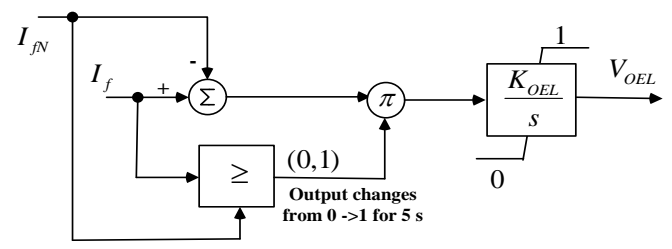

Fig. 7. OEL limiter.

\section{B. Overexcitation limiter implementation}

The system voltage reduction due to the switching operation or faults produces high reactive power requirements, by increasing the field current to maintain a constant generator voltage. In a remarkable system, the voltage drops, which may cause thermal overloading of the generator rotor winding if the generator voltage setpoint is not reduced within a certain time. In this situation, the OEL is automatically activated to limit the field current.

The purpose of the OEL is to limit the field current value to prevent the generator operating point from exceeding the field current limit and the rotor winding heating limit of Fig. 3 [30]-[32]. When the field current $I_{f}$ exceeds the pickup value $I_{f N}$, which is the nominal value of the field current, a positive signal will be supplied to the integrator, which enables the field current to attain a value of $1.5 I_{f N}$ for $5 \mathrm{~s}$. If the grid does not remove the disturbance after $5 \mathrm{~s}$, the OEL will decrease the field current to $1.05 I_{f N}$ as the permanent value, as shown in Fig. 7.

When the field current $I_{f}$ reduces below the pickup value, a negative signal will reset the integrator, which will provide a null error signal to the AVR summing point. The overexcited capability is limited by the rotor maximum excitation current (thermal limit). The response of OEL differs from that of the ceiling current limiter by the response value and response time.

\section{VHL limiter implementation}

The VHL limiter is activated to prevent the overflux in the machine, which occurs when the permissible ratio of the voltage to the frequency is exceeded by the following cases:

1. The terminal voltage of the generator rises to an impermissible value at a constant frequency.
2. The frequency drops to an impermissible value at a constant generator voltage.

3 . The frequency drops and the terminal voltage rises to an impermissible value

The input of the VHL limiter is the ratio of the per unit machine terminal voltage to the per unit speed. When the ratio exceeds a maximum threshold $(V / f)_{S P}$, shown here as 1.1 $\mathrm{pu}$, the difference is obtained by an interpolation function and is then integrated. The higher is the $(V / f)$ value, the faster the integrator will work. A negative signal $V_{V f}$ is sent to the AVR summing point. The negative signal reduces the voltage setpoint, and therefore, the SC output voltage decreases until the voltage to frequency ratio falls below the threshold.

When the difference becomes negative via the inequality block, the integrator is reset to zero to obtain null error signal becomes null, as shown in Fig. 8. Therefore, a $\mathrm{V} / \mathrm{Hz}$ will change the SC output voltage but will not have an effect on the machine frequency [33].

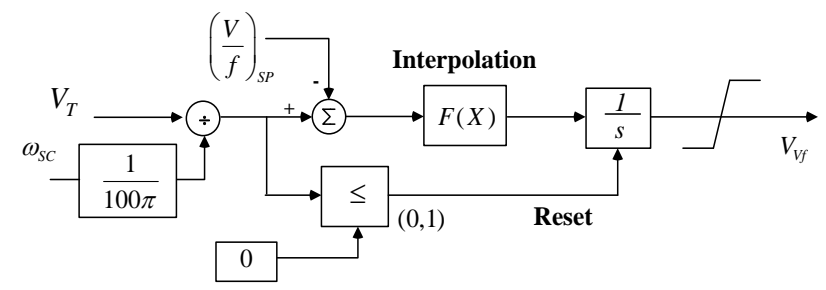

Fig. 8. VHL limiter.

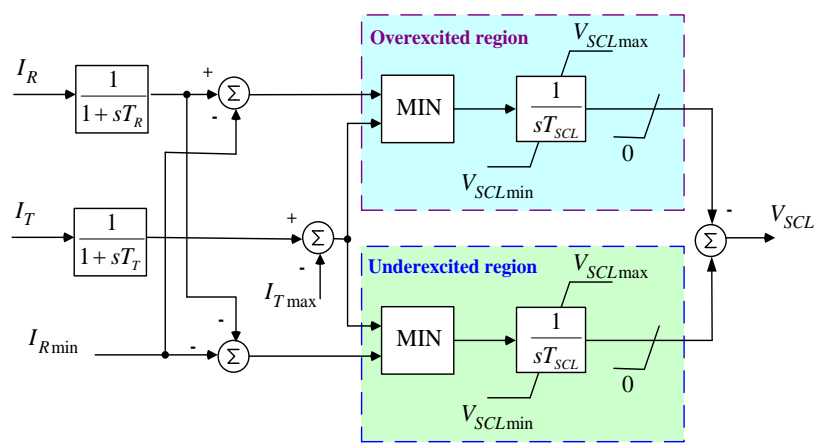

Fig. 9. SCL limiter.

\section{Stator current limiter implementation}

The SCL aims to ensure that the stator is operated within its ratings. Mathematically, the stator current can be decomposed into reactive and active components. This limiter action depends on the operating point, and SCL will control the reactive component to limit the absolute value of the stator current, which limits the losses (or heating $I^{2} R$ ) in the stator winding. In the overexcited region, the limiter needs to reduce the excitation current to reduce the absolute value of the stator current and thus declining the losses in the stator winding. Conversely, in the underexcited region, the excitation current needs to be increased to attain the allowable value which causes a decrease in the stator losses and heating. In the 
vicinity of a power factor of one, a further change of the terminal voltage will not decrease the stator current and is blocked by the parameter $I_{R \min }$.

The limiter is active when the stator current is higher than the pickup value $I_{T \max }$. If the difference is positive for more than $10 \mathrm{~s}$, the limiter is activated and worked, as shown in Fig. 9. This output is subtracted to the AVR summing point. When the $V_{S C L}$ is equal to 0 for more than $2 \mathrm{~s}$, the limiter is deactivated.

\section{SOFTWARE-IN-THE-LOOP SETUP}

The prospective future Western Danish power system run in the RTDS platform is driven by a MatLab script for system startup and disturbance simulations. The data of the system are collected by an OPC server and sent directly to the Matlab workspace. In MatLab, the signal is processed and evaluated by the GA objective. These steps are iterative by a closed loop with RTDS, OPC, and Matlab communications, as shown in Figs. 1 and 2. The loop will continue until the objective function is satisfied to determine the optimal parameters. This SiL simulation for parameter optimization is applied separately for POD and PID controller of the AVR system where GA objective functions are completely different.

\section{A. RTDS setup}

The whole DK1 system is modeled and compiled in RSCAD/Draft, and then run and monitored in RSCAD/Runtime. To run the system by a Matlab script, a communication connection is established between RSCAD/Runtime and Matlab. Once the connection is implemented, RSCAD/Runtime becomes a socket server and Matlab becomes a socket client. RSCAD/Runtime is commanded and run in real-time by a Matlab script via the TCP server [26]. In addition, the new parameter set is updated via this communication.

\section{B. OPC setup}

To collect the data from RTDS, a communication channel between RTDS with MatrikonOPC is setup with 3 steps, including an RTDS network channel, an RTDS network host, and an RTDS DNP3, which are described in [34]. First, the RTDS network channel is setup where the protocol is defined. Second, the RTDS network host is established inside the network channel. In the network host, the IP address of the GTNET card of the RTDS and the port number that have been defined in the GTNET card of the RTDS platform are filled. Last, the RTDS DNP3 is created to view the signals that are collected from the RTDS. After the communication is established, the RTDS and OPC can exchange data with each other. The measurement data are collected in the OPC server and sent to Matlab for the next steps.

\section{Matlab program}

A Matlab script commands and runs the system in realtime in RSCAD/Runtime via TCP communication. To acquire the data at the OPC server, a connection is setup in a
Matlab script to create a group object with defined properties. After obtaining the data and performing signal processing to convert to the proper format, the data are retrieved in the Matlab workplace for further analysis. When the data are sent to Matlab, OPC cleans up and disconnects to prepare for subsequent data collections.

With POD controller, frequency measurement is collected by OPC and sent to Matlab. Then a polynomial fitting is implemented to remove the fundamental frequency component. The oscillation component is analyzed by Prony analysis for extracting the frequency and damping ratio of the dominant oscillation mode. The damping ratio is maximized by GA objective function to determine the better parameters of POD and update the RTDS model for further verification.

The objective function of GA-based POD is the damping ratio maximization of the system oscillation mode, i.e.,

$$
f(x)=\max \left\{\xi=-\frac{\alpha}{\sqrt{\alpha^{2}+\beta^{2}}}\right\}
$$

where $\alpha$ and $\beta$ are real and imaginary parts of the dominant mode, respectively.

Meanwhile, with PID control of AVR, the objective function is the minimization of the difference of two AVR hardware and simulation outputs as follows:

$$
f(x)=\min \left(\sum\left(E_{f d s}-E_{f d h}\right)^{2}\right)
$$

where $E_{f d s}$ and $E_{f d h}$ are the outputs of the excitation with AVR simulation and that of hardware, respectively.

The parameter set is send to RSCAD/Runtime by a Matlab command for next iteration. These steps are continuously repeated until one of the termination parameters is achieved. The GA may be terminated after a certain number of generations when the objective value does not enhance after a certain generation. As a result, a near-optimal or optimal solution for parameter set is determined. The entire procedure is illustrated in Fig. 2.

\section{CASE STUDY}

To verify the parameter optimization for the PID controller of the AVR simulation model based on the GA, different tests, such as under-excitation and overexcitation tests and a load increase scenario, are investigated to compare the responses of the AVR hardware and simulation. In addition, a load increase scenario is investigated to evaluate the parameterization based on GA of the POD controller.

\section{A. AVR hardware test}

1) Underexcition and overexcitation condition test: The voltage of the grid side suddenly increases 0.1 pu at $t=0.75$ $\mathrm{s}$, SC immediately absorbs reactive power from the grid side to lower the voltage. The comparative results between AVR controller of HiL and that of simulation are shown in Fig. 10 with dash red curves for hardware and solid black ones for simulation.

As can be seen in Fig. 10(b), a natural response of SC rapidly absorbs reactive power to against the voltage increase 

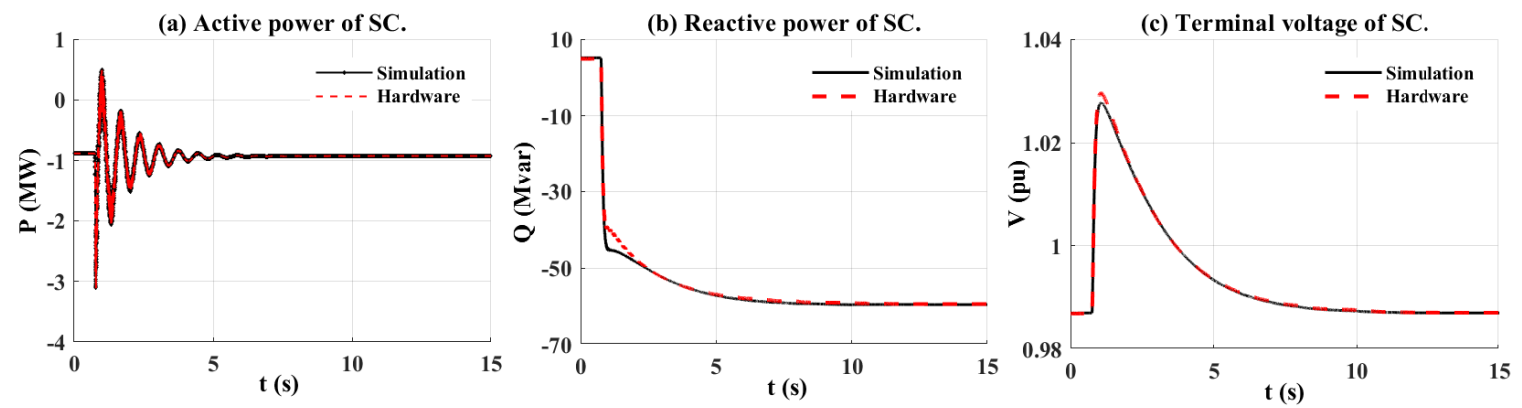

Fig. 10. Underexcitation responses. (a) Active power of SC. (b) Reactive power of SC. (c) Terminal voltage of SC.
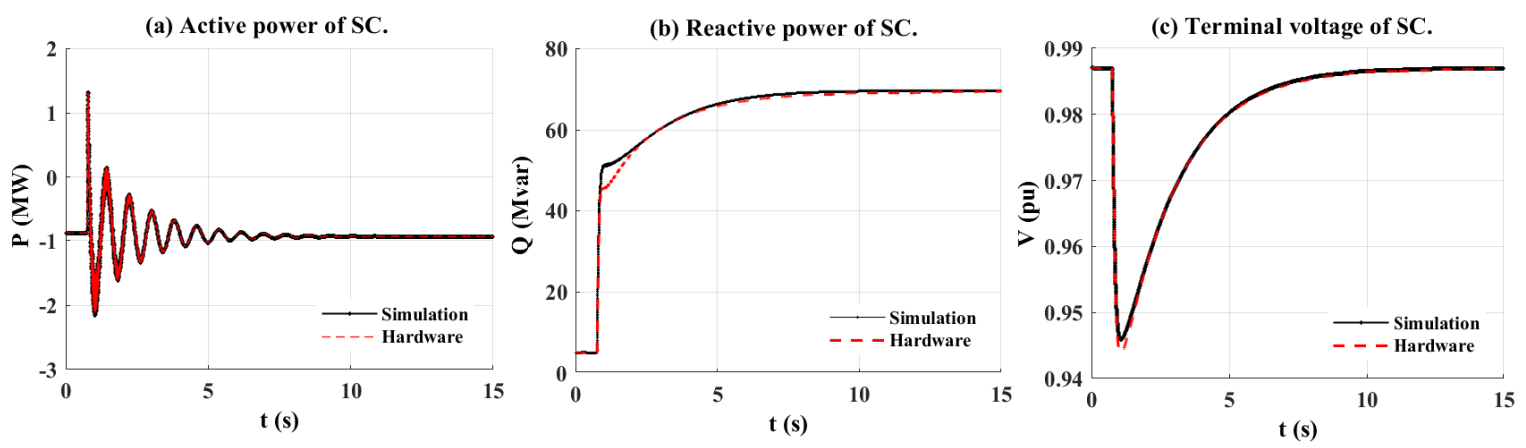

Fig. 11. Overexcitation responses of SC. (a) Active power of SC. (b) Reactive power of SC. (c) Terminal voltage of SC.
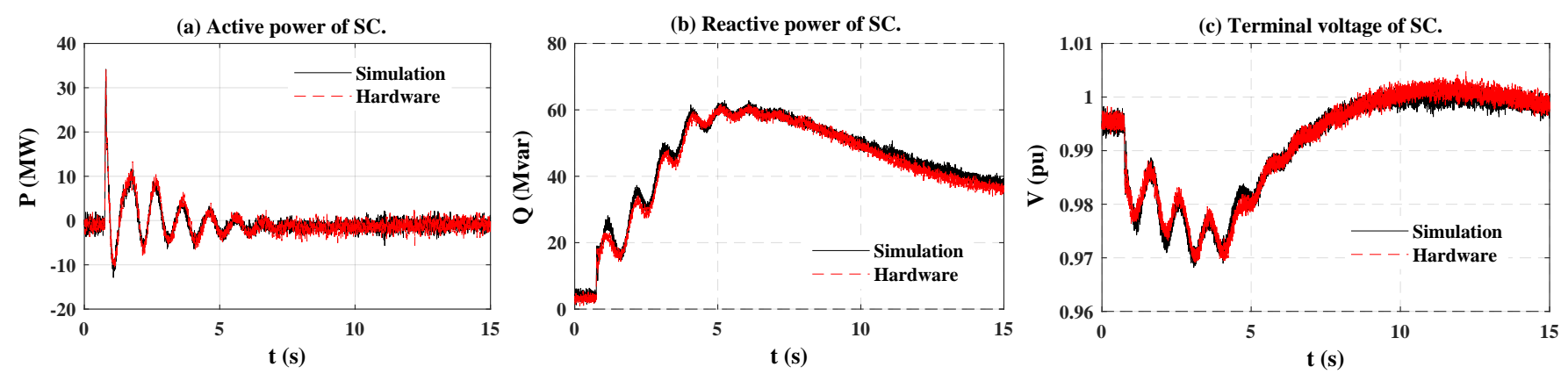

Fig. 12. SC responses during a load increase. (a) Active power of SC. (b) Reactive power of SC. (c) Terminal voltage of SC.

during transient period, AVR control following response to absorb more reactive power which lead to the voltage starts going down and gets back the pre-disturbance point.

It should be mentioned that AVR hardware control responds a bit slower than that of simulation due to the signal transferring time, which causes the terminal voltage of SC keep increasing a bit further 1.03 pu instead of 1.028 pu with that of simulation before getting the same new steady-state operating point after the disturbance.

A similar pattern can be observed in Fig. 11 with overexcitation condition when the grid voltage decrease $0.1 \mathrm{pu}$.

It is noteworthy that the active power responses of AVR hardware and simulation are almost similar because the AVR controller seems insensitive to the active power.

2) Load increase: Another test to verify the response of AVR hardware and simulation is investigated during $150 \mathrm{MW}$ load increase at $t=0.75 \mathrm{~s}$, as shown in Fig. 12. From the comparative results with simulation and hardware of AVR, the reactive power responses are similar for both cases. They quickly increase to counteract the voltage decrease and bring the terminal voltage back to the pre-disturbance point, increasing from around 4 Mvar to 61 Mvar and settling down at approximately 38 Mvar after around $10 \mathrm{~s}$. As a result, the terminal voltage response is similar for two AVR cases.

The active power responses are almost similar in terms of magnitude and settling time for two approaches. It can be concluded that parameter optimization based on SiL offers a parameter set for AVR simulation to have a similar behaviour to AVR hardware.

\section{B. POD parameterization test}

In order to verify the performance of SiL simulation for POD parameter optimization, a load increase disturbance is investigated in this section. Fig. 13 shows comparative results of system frequency, ROCOF, active power on transmission 


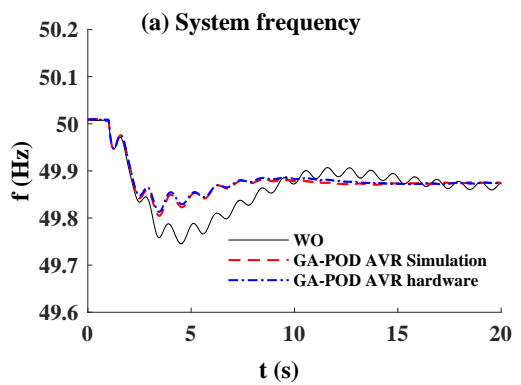

(d) Active power of SC

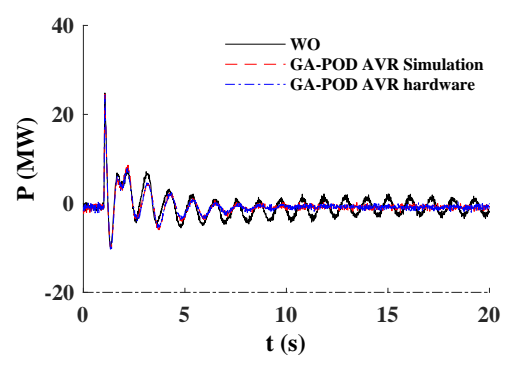

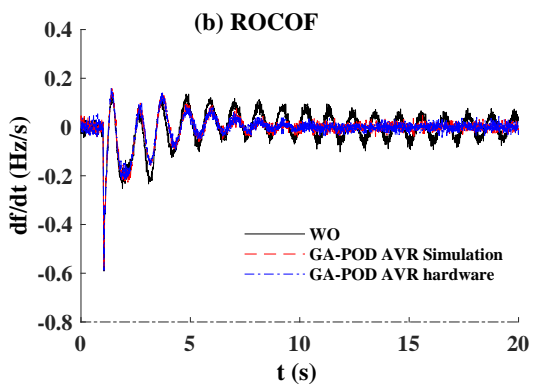

(e) Reactive power of SC

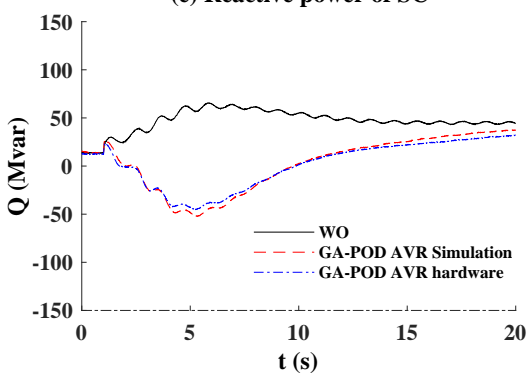

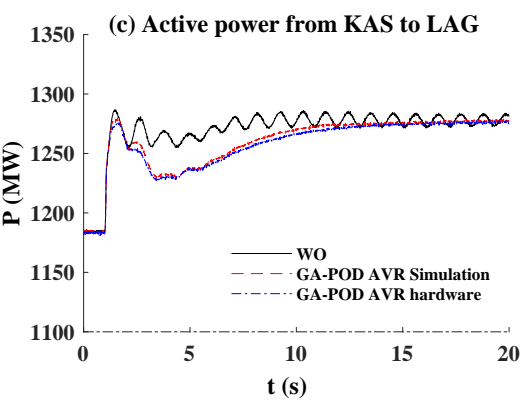

(f) Terminal voltage of SC

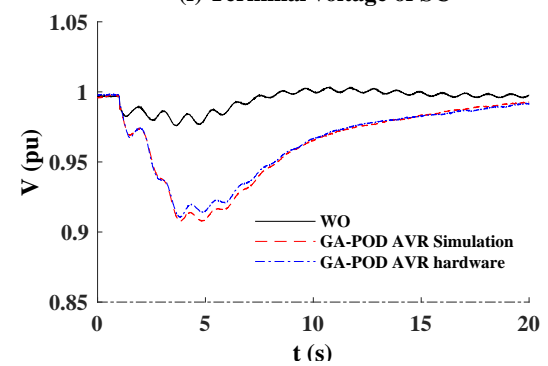

Fig. 13. Load increase disturbance: (a) System frequency. (b) ROCOF. (c) Active power from KAS to LAG. (d) Active power of SC. (e) Reactive power of SC. (f) Terminal voltage of SC.

lines, and SC responses WO in the solid line, with GAbased POD and AVR simulation in the dashed black red, with GA-based POD and AVR hardware in the dash-dot blue one, respectively. From the comparative results, it can be seen clearly that with POD controller the system response is significantly enhanced in terms of the damping ratio and frequency stability. As seen that the system frequency in Fig. 13(a), without POD it experiences a large and long oscillation (the dominant mode with $0.296 \%$ damping ratio) as well as a huge deviation $(0.25 \mathrm{~Hz})$ before getting a new equilibrium. On the contrary, with GA-based POD these parameters are significantly improved $14,5 \%$ of damping ratio and $0.18 \mathrm{~Hz}$ of the frequency deviation, respectively. By comparing ROCOF, a faster damping and a quicker settling down are obviously seen in Fig. 13(b) with POD controller.

An opposite trend is observed from the reactive power response of SC during the disturbance without and with POD controller. Instead of rapidly increasing the reactive power from around 13 Mvar to approximately 65 Mvar to keep the voltage constant at the nominal value as WO case, POD decreases the terminal voltage by absorbing around 58 Mvar reactive power (from 13 Mvar to around -45 Mvar) to control the power flow. Consequently, a large decrease and less oscillation are observed from the active powers on the transmission line from KAS to LAG with POD controller as shown in Fig. 13(c).

TABLE I

WITHOUT AND WITH GA COMPARISON OF DOMINANT MODE.

\begin{tabular}{c|c|c|c|c}
\hline Cases & Domi. mode & Freq (Hz) & Dam. ratio & Fre. peak $(\mathbf{H z})$ \\
\hline \hline WO & $-0.037+j 5.79$ & 0.919 & 0.00296 & 49.75 \\
\hline WGA & $-0.998+j 5.96$ & 0.953 & 0.145 & 49.82 \\
\hline
\end{tabular}

As expected, the active power of SC rapidly supports kinetic energy and quickly settles down with POD controller as seen in Fig. 13(d). As a result, the power oscillation and frequency stability are significantly improved during the disturbance with POD controller.

The parameters shown in Table I summarize the Prony analysis of the dominant mode with a significant enhancement of the system with POD controller based on nonlinear optimization algorithms. It can be concluded that with parameterization, the POD controller performs a significant enhancement in damping oscillation and frequency stability.

It is worth to mention that the system response is similar for AVR hardware and that of simulation. It can be concluded that parameter optimization based on SiL satisfies the optimization objective.

\section{CONCLUSION}

The paper firstly presents a HiL test for the AVR of SC before the deployment in the field. Different tests such as underexcitation and overexcitation conditions are implemented for AVR hardware which demonstrates the possible applications of HiL to validate and adjust the control function of the equipment in steady state and dynamics conditions before final construction implementation with lower cost, higher efficiency, and more flexibility.

Furthermore, a SiL simulation using EMT simulation for parameter optimization based on a nonlinear optimization tool of POD and PID controllers is proposed to offer a near-optimal or optimal solution for the control parameter set which can help control designers saving a lot of time. GA is selected here since it is a typical approach for tackling complex optimization problems. This SiL simulation can also solve different issues in power systems, where the RTDS is replaced by the real system, OPC represents the data acquisition system, and Matlab acts as the control center. 


\section{ACKNOWLEDGMENT}

This work is supported by Synchronous Condenser Application (SCAPP) project funded by ForskEL program, grant no. 12196 administrated by Energinet.dk.

\section{REFERENCES}

[1] L. Fan and Z. Miao, "Wind in Weak Grids: $4 \mathrm{~Hz}$ or $30 \mathrm{~Hz}$ Oscillations?" IEEE Trans. Power Systems, vol. 33, no. 5, pp. 5803-5804, Sept. 2018.

[2] S. Huang, J. Schmall, J. Conto, J. Adams, Y. Zhang and C. Carter, "Voltage control challenges on weak grids with high penetration of wind generation: ERCOT experience," in Proc. 2012 IEEE Power and Energy Society General Meeting, San Diego, CA, Jul. 22-26, 2012, pp. 1-7.

[3] L. Fan and Z. Miao, "An Explanation of Oscillations Due to Wind Power Plants Weak Grid Interconnection," IEEE Trans. Sustainable Energy, vol. 9, no. 1, pp. 488-490, Jan. 2018.

[4] M. S. Almas and L. Vanfretti, "RT-HIL testing of an excitation control system for oscillation damping using external stabilizing signals," in Proc. 2015 IEEE Power \& Energy Society General Meeting, Denver, CO, Jul. 26-30, 2015, pp. 1-5.

[5] H. T. Nguyen, G. Y. Yang, A. H. Nielsen, and P. H. Jensen, "Hardwarein-the-loop test for automatic voltage regulator of synchronous condenser," in Proc. 20th International Conference on Power Systems and Energy Conversion, Tokyo, Japan, Mar. 27-28, 2018, pp. 1-6.

[6] J. G. Kim, S. K. Kim, M. Park, and I. K. Yu, "Hardware-in-the-Loop simulation for superconducting DC power transmission system," IEEE Trans. Applied Superconductivity, vol. 25, no. 3, pp. 1-4, Jun. 2015.

[7] D. A. Martinez Montana, D. F. Celeita Rodriguez, D. I. Clavijo Rey, and G. Ramos, "Hardware and software integration as a realist SCADA environment to test protective relaying control," IEEE Trans. Industry Applications, vol. PP, no. 99, pp. 1-10, Dec. 2017.

[8] J. Jia, G. Y. Yang, A. H. Nielsen, and P. R. Hansen, "Impact of VSC control strategies and incorporation of synchronous condensers on distance protection under unbalanced faults," IEEE Trans. Industrial Electronics, vol. 66, no. 2, pp. 1108-1118, Feb. 2019.

[9] J. Berge and R. K. Varma, "Design and development of a static VAR compensator for load compensation using real-time digital simulator and hardware simulation," in Proc.2007 Large Engineering Systems Conference on Power Engineering, Montreal, Quebec, Oct. 10-12, 2007, pp. 6-12.

[10] V. Tuominen, H. Reponen, A. Kulmala, S. Lu, and S. Repo, "Realtime hardware- and software-in-the-loop simulation of decentralized distribution network control architecture," IET Generation, Transmission \& Distribution, vol. 11, no. 12, pp. 3057-3064, Sep. 2017.

[11] F. Alvarez-Gonzalez, A. Griffo, B. Sen, and J. Wang, "Real-time hardware-in-the-loop simulation of permanent magnet synchronous motor drives under stator faults," IEEE Trans. Industrial Electronics, vol. 64, no. 9, pp. 6960-6969, Sep. 2017.

[12] H. M. Hasanien, "Design Optimization of PID Controller in Automatic Voltage Regulator System Using Taguchi Combined Genetic Algorithm Method," IEEE Systems Journal, vol. 7, no. 4, pp. 825-831, Dec. 2013.

[13] D. Devaraj and B. Selvabala, "Real-coded genetic algorithm and fuzzy logic approach for real-time tuning of proportional-integral - derivative controller in automatic voltage regulator system," IET Generation, Transmission \& Distribution, vol. 3, no. 7, pp. 641-649, July 2009.

[14] D. Chitara, K. R. Niazi, A. Swarnkar and N. Gupta, "Cuckoo Search Optimization Algorithm for Designing of a Multimachine Power System Stabilizer," IEEE Trans. Industry Applications, vol. 54, no. 4, pp. 30563065, July-Aug. 2018.

[15] R. Jalayer and B. Ooi, "Co-Ordinated PSS Tuning of Large Power Systems by Combining Transfer Function-Eigenfunction Analysis (TFEA), Optimization, and Eigenvalue Sensitivity," IEEE Trans. Power Systems, vol. 29, no. 6, pp. 2672-2680, Nov. 2014.

[16] E. L. Miotto, P. B. de Araujo, E. de Vargas Fortes, B. R. Gamino and L. F. B. Martins, "Coordinated Tuning of the Parameters of PSS and POD Controllers Using Bioinspired Algorithms,' IEEE Trans. Industry Applications, vol. 54, no. 4, pp. 3845-3857, July-Aug. 2018.

[17] M. A. Abido, "Optimal design of power-system stabilizers using particle swarm optimization," IEEE Trans. Energy Conversion, vol. 17, no. 3, pp. 406-413, Sept. 2002.

[18] A. Moeini and I. Kamwa, "Analytical Concepts for Reactive Power Based Primary Frequency Control in Power Systems," IEEE Trans. Power Systems, vol. 31, no. 6, pp. 4217-4230, Nov. 2016.
[19] D. Rerkpreedapong, A. Hasanovic, and A. Feliachi, "Robust load frequency control using genetic algorithms and linear matrix inequalities," IEEE Trans. Power Systems, vol. 18, no. 2, pp. 855-861, May 2003.

[20] A. Safari, H. A. Shayanfar, and A. Kazemi, "Robust PWMSC damping controller tuning on the augmented Lagrangian PSO algorithm," IEEE Trans. Power Systems, vol. 28, no. 4, pp. 4665-4673, Nov. 2013.

[21] S. Q. Yuan and D. Z. Fang, "Robust PSS parameters design using a trajectory sensitivity approach," IEEE Trans. Power Systems, vol. 24, no. 2, pp. 1011-1018, May 2009.

[22] S. M. Baek and J. W. Park, "Nonlinear parameter optimization of FACTS controller via real-time digital simulator," IEEE Trans. Industry Appl., vol. 49, no. 5, pp. 2271-2278, Sep.-Oct. 2013.

[23] W. Qiao, G. K. Venayagamoorthy, and R. G. Harley, "Real-time implementation of a STATCOM on a wind farm equipped with doubly fed induction generators," IEEE Trans. Ind. Appl., vol. 45, no. 1, pp. 98-107, Jan./Feb. 2009.

[24] Synchronous Condensers Application in Low Inertia Systems (SCAPP), 2014. [online]. Available: http://www.scapp.dk/

[25] H. T. Nguyen, G. Y. Yang, A. H. Nielsen, and P. H. Jensen, "Frequency stability enhancement for low inertia systems using synthetic inertia of wind power," in Proc. 2017 IEEE Power \& Energy Society General Meeting, Chicago, IL, Jul. 16-21, 2017, pp. 1-5.

[26] RTDS, "RTDS hardware manual," Jan. 2009. [online]. Available: https://www.rtds.com/

[27] IEEE Std 421.5 system models for power system stability studies," New York, USA, Apr. 2006.

[28] IEEE Task Force on Excitation Limiters, "Underexcitation limiter models for power system stability studies," IEEE Trans. Energy Conversion, vol. 10, no. 3, pp. 524-531, Sep. 1995.

[29] G. Benmouyal, "The impact of synchronous generators excitation supply on protection and relays," Journal of Reliable Power, vol. 3, no. 1, pp. 1-16, Mar. 2012.

[30] G. K. Girgis and H. D. Vu, "Verification of limiter performance in modern excitation control systems," IEEE Trans. Energy Conversion, vol. 10, no. 3, pp. 538-542, Sep. 1995.

[31] IEEE Task Force on Excitation Limiters, "Recommended models for overexcitation limiting devices,", IEEE Trans. Energy Conversion, vol. 10, no. 4, pp. 706-713, Dec. 1995.

[32] A. Murdoch, G. E. Boukarim, B. E. Gott, M. J. D’Antonio, and R. A. Lawson, "Generator over excitation capability and excitation system limiters," in Proc. 2001 IEEE Power Engineering Society Winter Meeting, Columbus, OH, vol.1, pp. 215-220, Jan. 28-Feb. 1, 2001.

[33] A. Murdoch, R. W. Delmerico, S. Venkataraman, R. A. Lawson, J. E. Curran, and W. R. Pearson, "Excitation system protective limiters and their effect on volt/VAr control-design, computer modeling, and field testing," IEEE Trans. Energy Conversion, vol. 15, no. 4, pp. 440-450, Dec. 2000.

[34] Q. Wu, S-T. Cha, A. Saleem, and J. Østergaard, "Communication test for 'MatrikonOPC server for SCADA DNP 3' with RTDS,' Tech. Rep. 2010 .

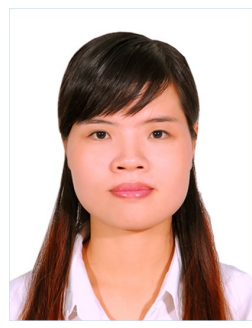

Ha Thi Nguyen received the B.Sc., M.Sc. and Ph.D. degrees in electric power systems from University of Science and Technology - the University of Danang, Vietnam in 2010, National Cheng Kung University, Taiwan in 2014 and Technical University of Denmark (DTU), Denmark in 2018, respectively. In 2017, she was a Visiting Scholar at the Center Energy Research, University of California San Diego, USA. Currently, she is working as a Postdoc at the Center for Electric Power and Energy at DTU. Her research interests are power system operation and control, real-time and hardware-in-the-loop simulation, frequency stability and control, and renewable energy integration. 


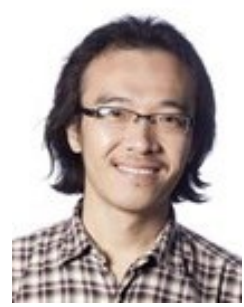

Guangya Yang received the B.E., M.E., and Ph.D. degrees all in the field of electric power system, in 2002, 2005, and 2008, respectively. Since 2009, he has been with the Technical University of Denmark, Kongens Lyngby, Denmark, as a Postdoctoral Researcher, and he is currently an Associate Professor with the Center for Electric Power and Energy, Department of Electrical Engineering, Technical University of Denmark. Since 2009, he has been leading several industrial collaborative projects in Denmark in the field of monitoring, operation and protection of renewable energy systems. His research interests include renewable energy integration, smart grids, and cyber-physical energy systems

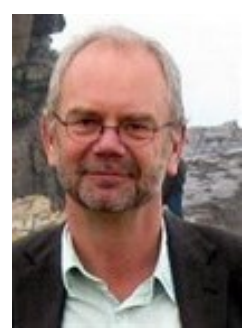

system.

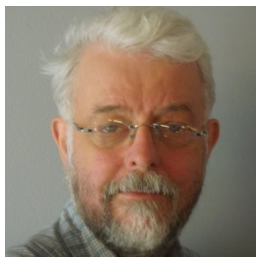

Arne Hejde Nielsen is an Associate Professor at the Centre for Electric Power and Energy, Department of Electrical Engineering, Technical University of Denmark, Kongens Lyngby, Denmark. He has 30 years experience in electric power engineering; the first years were from ASEA AB, Central Research and Development Department, Sweden, with focus on measurement technology and motor design and control. Over the past decade, his focus has been on electric power systems, especially on the implementation of renewable energy sources in the power

Peter Højgaard Jensen is a System Specialist at Siemens A/S. He received the M.Sc. degree in electrical engineering from the Technical University of Denmark, Lyngby in 1979. He has 34 years' experience from Danish Power Plants in maintenance, operation, management, engineering, power plant erection and commissioning. Five years in Siemens have been used for developing new control concept for Synchronous Condensers (SynCon's) and commissioning of 13 SynCon's in Denmark, Norway, Texas and California (175-270 MVAr). 